\title{
THE ARACHNIDA OF ST. CROIX, V. I.
}

\section{BY HARRY A. BEATTY}

This check-list of the ectoparasites occurring on mammals, birds and reptiles of St. Croix, V. I., holds no pretense for completness but the material at hand is sufficiently representative to gain our interest, and especially is this true since the presence of these parasites have remained unrecorded from the island. All species mentioned here were taken off of their natural hosts and the determinations were made by H. E. Eving, excepting the half-engorged nymphs of Amblyomma which were determined by C. N. Smith. This being the first reference to Amblyomma on St. Croix it may have arrived here quite recently, possibly from Antigua, B.W.I. where it. is reported as common.

To encompass a complete faunal report on St. Croix it should be mentioned here that a large collection of spiders was sent to Elizabeth B. Bryant and her paper entitled "Notes on the Spiders of the Virgin Islands" appeared in the "Bulletin of the Museum of Comparative Zoology," Vol. LXXXIX, No. 7, 1942.

Order ACarrna

Suborder Astighata

SARCOPTIDAE

\section{Notoedres cati Hering}

Off domestic cat.

2 Cnemidocoptes sp.

Known as "scaly legs" of forrls.

3 Sarcoptes scabiei canis Gerlach

Off dog.

4 Psoroptes communis Furstenberg

Taken off cattle.

\section{ANALGESIDAE}

1 Analgesidae gen \& sp.

Off Red-tailed Hawk (Buteo jamaicensis), and Bare-legged Orl (Otus nudipes newtoni).

2 Analgesidae gen \& sp.

Taken off Ani (Crotophaga ani), Mocker (Mimus polyglottos orpheus), Thrasher (Margarops f. fuscatus), Yellow warbler (Dendroica petechia cruciana). 
3 Analgesidae gen \& sp.

Takon off Oyster-catcher (Haematopus ostralegus palliatus). 4 Analgesidae gen \& sp.

Taken off Ground-Dove (Columbigallina passerina nigrirostris), Hummingbird (Sericoles; $h$. holosericeus), Kingbird (Tyrannus $d$. dominicensis).

5 Eupterolichus sp.

Off Ani (Crotophaga ani).

6 Falculifer sp.

Off Scaled Pigeon (Columba squamosa).

7 Pterodectes sp.

Off Mocker (Mimus polyglottus orpheus).

Suborder Brach YPODA

DEMODICIDAE

1 Demodex canis Leydig

Taken off dog.

GHQEYLETIDAE

1 Cheyletus sp.

Off domestic fowl.

Suborder Mesostigmata

DERMANYSSIDAE

1 Liponyssus bursa Berlese

Off domestic forvl.

PARASITIDAE

1 Parasitidae gen \& sp.

Taken off a beetle, Metamasius hemipterus.

2 Echinolaelaps echidninus Berlese

Off gray Rat, Rattus rattus alexandrinus.

3 Periglischrus sp.

Off leaf-nosed bat, Artibeus $j$. jamaicensis.

4 Gekobia. sp.

Taken from the abdomen of a gecko, Hemidactylus mabouia

5 Macrocheles sp.

Clustered about the thorax of beetle, Cerambycidae sp. ..

6 Galumna sp.

Collected from an old bird nest. 
IXODIDAT

1 Ixodidae gen \& sp.

Taken off Bat, Noctilio leporinus mastivus. Taken off Mongoose, Herpestes birmanicus.

2 Haemaphysalis chordeiles Packard

Taken off Wilson Plover (Charadrius wilsonia).

3 Dermacentor nitens Neumann

A common parasite of the Horse (Equus caballus) and Donkey (Equus asinus). Rarely found on the deer (Odocoileus virginianus).

4 Rhipicephalus sanguineus Latreille

Common, parasite of dogs.

5 Boophilus annulatus microplus Canestrini

Abundant parasite of deer (Odocoileus virginionus), and cattle. Rare on sheep and goats.

6 Amblyomma sp.

Det. G. N. Smith. Nymph taken off man. May 1935. 